\title{
Article \\ Ceftazidime-Avibactam for the Treatment of Multidrug-Resistant Pathogens: A Retrospective, Single Center Study
}

\author{
Maria Di Pietrantonio ${ }^{1}$, Lucia Brescini ${ }^{1,2, *}$, Jennifer Candi ${ }^{3}$, Morroni Gianluca ${ }^{2}$ D, Francesco Pallotta ${ }^{1,3}$, \\ Sara Mazzanti ${ }^{1,2}$, Paolo Mantini 1,3, Bianca Candelaresi 1,3, Silvia Olivieri 1,3 , Francesco Ginevri 1,3, \\ Giulia Cesaretti ${ }^{1,3}$, Sefora Castelletti ${ }^{4}$, Emanuele Cocci ${ }^{5}$, Rosaria G. Polo ${ }^{5}$, Elisabetta Cerutti ${ }^{6}$, \\ Oriana Simonetti ${ }^{7}$, Oscar Cirioni ${ }^{1,2}$, Marcello Tavio ${ }^{4}$, Andrea Giacometti ${ }^{1,2}$ and Francesco Barchiesi ${ }^{2,8}$
}

\section{check for} updates

Citation: Di Pietrantonio, M.

Brescini, L.; Candi, J.; Gianluca, M.; Pallotta, F.; Mazzanti, S.; Mantini, P.; Candelaresi, B.; Olivieri, S.; Ginevri, F.; et al. Ceftazidime-Avibactam for the Treatment of Multidrug-Resistant Pathogens: A Retrospective, Single Center Study. Antibiotics 2022, 11, 321 https://doi.org/10.3390/ antibiotics 11030321

\section{Academic Editor: Mehran Monchi}

Received: 2 February 2022

Accepted: 23 February 2022

Published: 28 February 2022

Publisher's Note: MDPI stays neutral with regard to jurisdictional claims in published maps and institutional affiliations.

Copyright: (C) 2022 by the authors. Licensee MDPI, Basel, Switzerland. This article is an open access article distributed under the terms and conditions of the Creative Commons Attribution (CC BY) license (https:// creativecommons.org/licenses/by/ $4.0 /)$.
1 Infectious Diseases Clinic, Ospedali Riuniti Umberto I, Via Conca 71, 60126 Ancona, Torrette, Italy; maria.dipietrantonio@ospedaliriuniti.marche.it (M.D.P.); pallottafrancesco1993@gmail.com (F.P.); sara.mazzanti@hotmail.it (S.M.); paolomantini90@gmail.com (P.M.); b.candelaresi@gmail.com (B.C.); silviaolivieri1992@gmail.com (S.O.); fginevri@hotmail.it (F.G.); giuliacesaretti@hotmail.it (G.C.); o.cirioni@univpm.it (O.C.); andrea.giacometti@ospedaliriuniti.marche.it (A.G.)

2 Department of Biomedical Sciences and Public Health, Polytechnic University of Marche Medical School, Via Tronto 10/a, 60020 Ancona, Torrette, Italy; g.morroni@pm.univpm.it (M.G.); f.barchiesi@staff.univpm.it (F.B.)

3 Faculty of Medicine, Polytechnic University of Marche Medical School, Via Tronto 10/a, 60126 Ancona, Torrette, Italy; jennifer.candi@gmail.com

4 Infectious Diseases, Ospedali Riuniti Umberto I, Via Conca 71, 60126 Ancona, Torrette, Italy; seforacastelletti@gmail.com (S.C.); marcello.tavio@ospedaliriuniti.marche.it (M.T.)

5 Hospital Pharmacy, Ospedali Riuniti Umberto I, Via Conca 71, 60126 Ancona, Torrette, Italy; emanuele.cocci@ospedaliriuniti.marche.it (E.C.); rosariagerarda.polo@ospedaliriuniti.marche.it (R.G.P.)

6 Anesthesia and Transplant Surgical Intensive Care Unit, Ospedali Riuniti Umberto I, Via Conca 71, 60126 Ancona, Torrette, Italy; elisabetta.cerutti@ospedaliriuniti.marche.it

7 Clinic of Dermatology, Department of Clinical and Molecular Sciences, Polytechnic University of Marche, Via Conca 71, 60126 Ancona, Torrete, Italy; o.simonetti@univpm.it

8 Infectious Diseases Unit, Azienda Ospedaliera Ospedali Riuniti Marche Nord, 61122 Pesaro, Pesaro and Urbino, Italy

* Correspondence: 1.brescini@staff.univpm.it; Tel.: +39-071-596-3502

Abstract: Background: Ceftazidime/avibactam is a new cephalosporin/beta-lactamase inhibitor combination approved in 2015 by the FDA for the treatment of complicated intra-abdominal and urinary tract infection, hospital-acquired pneumoniae and Gram-negative infections with limited treatment options. Methods: In this retrospective study, we evaluate the efficacy of ceftazidime/avibactam treatment in 81 patients with Gram-negative infection treated in our center from January 2018 to December 2019. The outcome evaluated was 30-days survival or relapse of infection after the first positive blood culture. Results: the majority of patients were 56 male (69\%), with median age of 67 . Charlson's Comorbidity Index was $>3$ in 58 patients. In total, $46 \%$ of the patients were admitted into the medical unit, $41 \%$ in the ICU, and $14 \%$ in the surgical ward. Of the patients, $78 \%$ had nosocomial infections, and $22 \%$ had healthcare-related infections. The clinical failure rate was 35\%: 13 patients died within 30 days from the onset of infection. The outcome was influenced by the clinical condition of the patients: solid organ transplantation $(p=0.003)$ emerged as an independent predictor of mortality; non-survival patients most frequently had pneumonia $(p=0.009)$ or mechanical ventilation $(p=0.049)$. Conclusion: Ceftazidime-avibactam showed high efficacy in infections caused by MDR Gram-negative pathogens with limited therapeutic options.

Keywords: Gram-negative pathogens; multidrug resistance; ceftazidime-avibactam 


\section{Introduction}

In 2017, the WHO published a list of antibiotic-resistant priority pathogens, a catalogue of 12 species of bacteria that pose the greatest threat to human health: the list is divided in 3 categories (critical, high and medium priority) according to the urgency of the need for new antibiotics.

The critical priority category includes multidrug-resistant Gram-negative bacteria (Acinetobacter baumannii, Pseudomonas aeruginosa, and various Enterobacteriaceae), especially E. coli and K. pneumoniae which are the most involved species in blood stream infections (BSIs) and a cause of concern due to the wide antibiotic resistance patterns [1,2].

Combination therapy seems to be more successful than monotherapy for the treatment of MDR Gram-negative infections (i.e., colistin-polimixin B or tigecycline in combination with a carbapenem) and could reduce the insurgence of antibiotic resistance. Indeed, colistin should be used in combination therapy to avoid the selection of resistant strains (CRE). New therapeutic options include the $\beta$-lactam- $\beta$-lactamase inhibitor combination ceftazidime-avibactam (CZA), used in monotherapy or combination with aztreonam [3]. CZA couples a well-known cephalosporin with a novel non- $\beta$-lactam $\beta$-lactamase inhibitor. Avibactam inhibits ESBLs, AmpC $\beta$-lactamases (expressed in Pseudomonas aeruginosa and Enterobacteriaceae), class A carbapenemases (including the Klebsiella pneumoniae carbapenemase KPC) and OXA-48 $\beta$-lactamase family $[4,5]$.

CZA is indicated for the treatment of complicated intra-abdominal infections (cIAI), complicated urinary tract infections (cUTI), hospital-acquired pneumonia (HAP) including VAP, and infections due to Gram-negative organisms with limited treatment options. The recommended intravenous dose in adults with creatinine clearance $>50$ is 2 g every $8 \mathrm{~h}$ [6-9]. Recent studies demonstrated that CZA was a promising drug for the treatment of severe KPC-producing K. pneumoniae (KPC-Kp) and reduced the mortality in BSIs patients [10-12].

The aim of this retrospective, observational study was to evaluate the efficacy of CZA administered in Gram-negative infections at a university hospital located in Central Italy.

\section{Results}

During the study period, a total of 81 patients received CZA therapy. Baseline characteristics of the patients included in the study are shown in Table 1 . The majority were male $(69 \%)$ with a median age of 67 years. Ninety-four percent of patients presented comorbidity, the most frequent being cardiovascular, renal and neurological diseases $(67 \%, 30 \%$ and $28 \%$, respectively). The Median Charlson Comorbidity Index was 5.

Table 1. Demographic and clinical characteristics of the study cohort.

\begin{tabular}{ccccc}
\hline & All $(n=81)$ & $\begin{array}{c}\text { Successful Clinical } \\
\text { Outcome }(n=52)\end{array}$ & $\begin{array}{c}\text { Clinical Failure } \\
(n=29)\end{array}$ \\
\hline Variables & & & & \\
\hline Patients variables & & & & \\
\hline Sex & & & & \\
\hline Male & $56(69 \%)$ & $39(75 \%)$ & $17(59)$ & 0.126 \\
\hline Female & $25(31 \%)$ & $13(25 \%)$ & $12(41 \%)$ & $67(58-75)$ \\
\hline Charlson's Comorbidity Index $\geq 3$ & $67(56-75)$ & $67(55.75-75.25)$ & $24(83 \%)$ & 0.121 \\
\hline
\end{tabular}


Table 1. Cont.

\begin{tabular}{|c|c|c|c|c|}
\hline & All $(n=81)$ & $\begin{array}{l}\text { Successful Clinical } \\
\text { Outcome }(n=52)\end{array}$ & $\begin{array}{l}\text { Clinical Failure } \\
\quad(n=29)\end{array}$ & $p$ \\
\hline \multicolumn{5}{|l|}{ Comorbidities } \\
\hline Diabetes & $17(21 \%)$ & $9(17 \%)$ & $8(27 \%)$ & 0.276 \\
\hline COPD & $7(9 \%)$ & $4(8 \%)$ & $3(10 \%)$ & 0.697 \\
\hline Hematological malignancies & $11(4 \%)$ & $7(14 \%)$ & $4(14 \%)$ & 1 \\
\hline Solid tumors & $17(21 \%)$ & $9(17 \%)$ & $8(28 \%)$ & 0.278 \\
\hline Chronic Hepatitis & $15(19 \%)$ & $9(17 \%)$ & $6(21 \%)$ & 0.707 \\
\hline Cardiovascular Disease & $54(67 \%)$ & $37(71 \%)$ & $17(59 \%)$ & 0.251 \\
\hline Neurological disease & $22(28 \%)$ & $13(25 \%)$ & $9(32 \%)$ & 0.495 \\
\hline Chronic kidney disease & $24(30 \%)$ & $14(27 \%)$ & $10(35 \%)$ & 1 \\
\hline HIV & $2(3 \%)$ & $1(2 \%)$ & $1(3 \%)$ & 1 \\
\hline Neutropenia & $2(3 \%)$ & $1(2 \%)$ & $1(3 \%)$ & 1 \\
\hline Gastrointestinal disease & $15(19 \%)$ & $9(17 \%)$ & $6(21 \%)$ & 0.707 \\
\hline SOT & $8(10 \%)$ & $1(2 \%)$ & $7(24 \%)$ & 0.003 \\
\hline \multicolumn{5}{|l|}{ Wards submitting index culture } \\
\hline Intensive care unit & $33(41 \%)$ & $19(37 \%)$ & $14(48 \%)$ & 0.320 \\
\hline Surgery & $11(14 \%)$ & $10(19 \%)$ & $1(3.4 \%)$ & 0.04 \\
\hline Medicine & $37(46 \%)$ & $23(44 \%)$ & $14(48 \%)$ & 0.726 \\
\hline \multicolumn{5}{|l|}{ Pre-infection variables } \\
\hline Central venous catheter & $55(68 \%)$ & $34(64 \%)$ & $21(72 \%)$ & 0.223 \\
\hline Nasogastric tube & $5(6 \%)$ & $2(4 \%)$ & $3(10 \%)$ & 0.244 \\
\hline Surgical drainage & $13(16 \%)$ & $8(15 \%)$ & $5(17 \%)$ & 1 \\
\hline Bladder catheter & $55(68 \%)$ & $34(65 \%)$ & $21(72 \%)$ & 0.516 \\
\hline Endoscopy $^{\mathrm{a}}$ & $4(5 \%)$ & $3(6 \%)$ & $1(3 \%)$ & 1 \\
\hline Mechanical ventilation $^{a}$ & $11(14 \%)$ & $4(8 \%)$ & $7(24 \%)$ & 0.049 \\
\hline $\mathrm{CVVH}$ & $13(16 \%)$ & $6(12 \%)$ & $7(24 \%)$ & 0.206 \\
\hline Steroid therapy ${ }^{b}$ & $27(33 \%)$ & $17(33 \%)$ & $10(35 \%)$ & 0.870 \\
\hline Immunosuppressive therapy $b, c$ & $14(17 \%)$ & $6(12 \%)$ & $8(28 \%)$ & 0.067 \\
\hline Previous surgery ${ }^{d}$ & $43(53 \%)$ & $30(58 \%)$ & $13(45 \%)$ & 0.266 \\
\hline \multicolumn{5}{|l|}{ Infection variables } \\
\hline Nosocomial infection & $63(78 \%)$ & $40(77 \%)$ & $23(80 \%)$ & 0.804 \\
\hline Polymicrobial infections & $31(38 \%)$ & $22(42 \%)$ & $9(31 \%)$ & 0.317 \\
\hline Septic shock & $16(20 \%)$ & $11(21 \%)$ & $5(17 \%)$ & 0.672 \\
\hline Pneumoniae & $66(82 \%)$ & $38(73 \%)$ & $28(97 \%)$ & 0.009 \\
\hline \multicolumn{5}{|l|}{ Sites of isolation } \\
\hline Urinary tract & $15(19 \%)$ & $10(19 \%)$ & $5(17 \%)$ & 0.825 \\
\hline Bronchial/pleural fluid & $27(33 \%)$ & $16(31 \%)$ & $11(38 \%)$ & 0.512 \\
\hline abdominal fluid & $5(6 \%)$ & $2(4 \%)$ & $3(10 \%)$ & 0.343 \\
\hline wounds & $6(7 \%)$ & $6(12 \%)$ & 0 & 0.083 \\
\hline blood & $23(28 \%)$ & $16(31 \%)$ & $7(24 \%)$ & 0.526 \\
\hline
\end{tabular}


Table 1. Cont.

\begin{tabular}{|c|c|c|c|c|}
\hline & All $(n=81)$ & $\begin{array}{l}\text { Successful Clinical } \\
\text { Outcome }(n=52)\end{array}$ & $\begin{array}{l}\text { Clinical Failure } \\
\qquad(n=29)\end{array}$ & $p$ \\
\hline \multicolumn{5}{|l|}{ Pathogens } \\
\hline K. pneumoniae KPC & $64(79 \%)$ & $22(76 \%)$ & $32(62 \%$ & 0.225 \\
\hline P. aeruginosa & $10(12 \%)$ & $4(14 \%)$ & $6(12 \%)$ & 0.739 \\
\hline E. coli & $5(6 \%)$ & $3(10 \%)$ & $2(4 \%)$ & 0.534 \\
\hline Other ${ }^{\mathrm{e}}$ & $2(2 \%)$ & 0 & $2(4 \%)$ & 0.534 \\
\hline Empirical use & $6(7 \%)$ & 0 & $6(12 \%)$ & 0.08 \\
\hline \multicolumn{5}{|l|}{ Treatment variables } \\
\hline $\begin{array}{l}\text { Previous therapy with others } \\
\text { regimens } b, f\end{array}$ & $29(36 \%)$ & $18(35 \%)$ & $11(38 \%)$ & 0.729 \\
\hline $\begin{array}{l}\text { Days of antibiotic therapy } \\
\text { (median) }\end{array}$ & $11(7-14)$ & $10(7-14)$ & $13(7-14)$ & 0.419 \\
\hline Combination therapy & $50(62 \%)$ & $32(62 \%)$ & $18(62 \%)$ & \\
\hline
\end{tabular}

Data are expressed as No. (\%) unless otherwise specified. Abbreviations: IQR-interquartile range, COPDchronic obstructive pulmonary disease, SOT-solid organ transplantation, CVVH—continuous veno-venous hemofiltration. ${ }^{a}$ During the $72 \mathrm{~h}$ preceding BSI onset. ${ }^{\mathrm{b}}$ During the 30 days preceding BSI onset. ${ }^{\mathrm{c}}$ Excluding therapy with steroids. ${ }^{\mathrm{d}}$ During the 3 months preceding BSI onset. ${ }^{\mathrm{e}}$ Others: $1 \mathrm{~S}$. maltophilia and $1 \mathrm{~K}$. aerogenes. ${ }^{\mathrm{f}}$ Previous therapy: colistin plus tygecicline plus meropenem; 5 colistin plus meropenem; 4 colistin; 1 gentamicin plus tygecicline; 3 tygecicline plus meropenem; 2 gentamicin; 2 tygecicline; gentamicin plus colistin plus meropenem; 1 colistin plus tygecicline plus fosfomycin plus tygecicline; 2 cephalosporins plus fosfomycin; 1 cephalosporins; 1 quinolone; 2 quinolone plus meropenem; 1 ceftolozane-tazobactam; 2 meropenem.

Forty-six percent of patients were hospitalized in medical wards and forty-one percent in ICUs. Septic shock was present in $20 \%$ of the overall population and pneumoniae in $82 \%$ of patients. Fourteen percent of pneumoniae cases were ventilator associated. A high proportion of patients carried a central venous catheter (CVC) $(68 \%)$ and urinary catheter (CV) $(68 \%)$. Solid organ transplantation (SOT) was the most common type of surgery characterizing these patients ( $28 \%$ of patients who had undergone surgery). In our case, liver transplantation was the only type of SOT.

CZA was mainly prescribed in complicated infections with limited therapeutic options (46\%). The other cases of prescription included HAP/VAP (30\%), cUTI (15\%) and cIAI (9\%).

The most frequently isolated pathogens were K. pneumoniae in $79 \%$ of cases, P. aeruginosa in $12 \%$, and E. coli in $6 \%$. Other pathogens were isolated in $2 \%$ of the cases, while in $7 \%$ of the cases, CZA was administered as an empirical treatment. Thirty-eight percent of patients had mixed infection. The majority of K. pneumoniae strains were KPC producers (95\%). All strains were susceptible to CZA. The sites of isolation were bronchial secretions or pleural fluid $(33 \%)$, blood $(28 \%)$, urinary tract $(19 \%)$, wounds $(7 \%)$ and intra-abdominal fluid $(6 \%)$.

CZA was administered with other antibiotics in $62 \%$ of patients: $19 \%$ of cases with tigecycline, $16 \%$ with colistin, $12 \%$ with fosfomycin, $12 \%$ with gentamicin, $11 \%$ with meropenem and $4 \%$ with amikacin. The median time of ceftazidime-avibactam administration was 11 days (Figure 1). Moreover, 29 patients had received other antibiotics in the 30 days before the administration of CZA.

Sixty-four percent of patients achieved a successful outcome, while thirty-six percent of patients did not. Of these, 13 patients died within 30 days from the onset of infection (30-day crude mortality $16 \%$ ), while 16 patients presented an infection relapse (microbiological failure rate $21 \%$ ). Among them, 12 patients survived, while 4 died. The 12 surviving patients with infection relapse were treated with CZA (38\%) or with other therapy $(62 \%)$. 


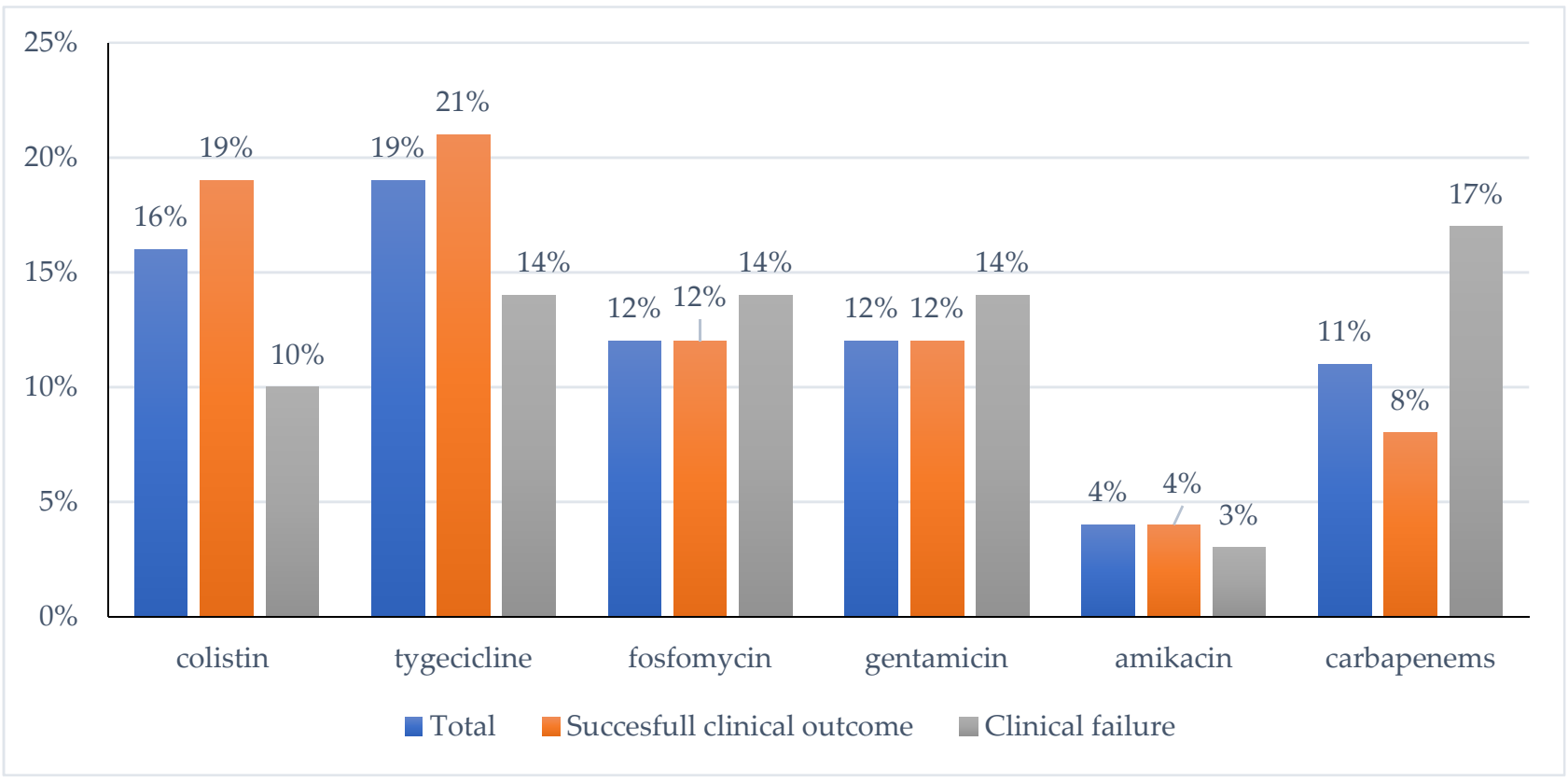

Figure 1. Clinical results of administration of CZA in combination therapy. The percentage showed refers to the total number of patients for each group (total, successful clinical outcome, and clinical failure).

A significantly higher proportion of patients with clinical failure received SOT $(p=0.003)$, mechanical ventilation $(p=0.049)$, or had pneumoniae $(p=0.009)$. Conversely, patients with successful clinical outcome were hospitalized more frequently in surgery wards $(p=0.04)$. No statistically significant differences were observed in treatment-related variables.

In the multivariate logistic regression analysis, only SOT emerged as independent predictors of failure treatment [OR $12.100(1.369-106.971), p=0.025]$.

\section{Discussion}

Infections caused by multidrug-resistant Gram-negative germs represent a major cause of mortality and a challenge for the physician. CZA is a new cephalosporin/beta-lactamase inhibitor combination approved in 2015 by the FDA for the treatment of complicated intraabdominal and urinary tract infection, hospital-acquired pneumoniae and Gram-negative infections with limited treatment options. In this study, we retrospectively evaluated the Gram-negative bacterial infections treated with CZA that occurred in the Ospedali Riuniti Umberto I Hospital, in the period between January 2018 and December 2019. The purpose of the study was to evaluate the efficacy of CZA and risk factors related to 30-day mortality in subjects treated with this antibiotic.

The clinical success achieved in patients treated with ceftazidime-avibactam was $64 \%$. In the literature, the success rate of the treatment ranges from $53 \%$ to $71 \%$ [10-12]. The variability is related to the different populations enrolled in the studies, the different species isolated, the site of the infections and the criteria used.

In our study, the infections treated with CZA were caused by several Gram-negative pathogens: the most isolated species was K. pneumoniae (79\%), followed by P. aeruginosa $(12 \%)$ and E. coli $(6 \%)$. Uncommon isolated Gram-negative were Stenotrophomonas maltophilia (1\%) and Klebsiella aerogenes (1\%). Furthermore, in 38\% of cases, the patients presented with polymicrobial infections. We observed a high relapse rate $(21 \%)$. This percentage was higher than the data seen in the literature, in which relapses of BSI due to KPC-Kp is around 8-9\% [10], while lower percentages were observed in infections other than BSIs (about 3-4\%) [12]. Conversely, we observed that BSIs had a lower relapse rate (20\%), compared to other infections (cUTI 25\%, cIAI 50\% and HAP/VAP 29\%). Possible explanations for the high number of relapses may be the heterogeneity of the therapies ad- 
ministered, the delay of start therapy with CZA, or the non-homogeneity of the pathogens isolated. In the clinical practice, antibiotic therapy is often remodeled according to the patient's clinical progress: worsening during the use of a therapeutic plan leads to a change in the chosen molecules, even if the duration of therapy is still shorter than that recommended. Additionally, therapy is often set empirically without waiting for the species identification and antibiotics susceptibility results.

According to other data described in the literature $[10,12,13]$, our study demonstrated that mechanical ventilation and pneumonia were correlated with higher 30-day mortality. Our results agree with one retrospective observational multicentric Italian study including 138 adults with KPC-Kp infections who received CZA salvage therapy. The authors compared the 30-days mortality in 104 patients with KPC-Kp bacteremia who received CZA and 104 patients with KPC-Kp BSIs that were managed with regimens excluding CZA. In a multivariate analysis, mechanical ventilation resulted in being statistically associated with 30-days mortality [10]. In another retrospective observational study recently published, pneumonia was a variable independently associated with 14-days mortality in 47 patients treated for $>72 \mathrm{~h}$ with CZA for KPC-Kp infections [13].

In our study, surgical patients showed greater clinical success with CZA therapy. In fact, in the most cases, these patients had a surgical site infection, a less serious clinical condition than patients admitted to medical and intensive care wards, and often underwent surgery to control the source of infection.

Interestingly, the only variable independently associated with the failure of CZA therapy at multivariate analysis was SOT. Carbapenem-resistant K. pneumoniae infection was an independent risk factor for mortality in liver transplantation recipients in some study [14-16] and is more frequent in these patients than in the general population, ranging from $6 \%$ to $23 \%$ [17-19]. Initially, few data were available in literature about the efficacy of CZA in patients with liver transplantations. In a recent study, Chen et al. evaluated the efficacy and safety of CZA in 21 patients infected by carbapenem-resistant K. pneumoniae after liver transplantation [20]. The 14-day and 30-day mortality rates were $28.6 \%$ and $38.1 \%$, respectively, consistent with other reports [10]. The fact that SOT represented an independent risk factor for a negative outcome can be caused by the frequent surgical complications, long hospitalization and polymicrobial infections observed in this subgroup of patients.

\section{Methods}

The setting was the 980-bed Regional University Hospital in Ancona, tertiary referral center. A cohort of 81 patients, treated with at least $72 \mathrm{~h}$ of CZA therapy and who were $\geq 18$ years old, with a Gram-negative infection diagnosed between January 2018 and December 2019, was considered: $46 \%$ of them were admitted in the medical unit, $41 \%$ in the ICU, and $14 \%$ in surgical wards.

Patient variables included age, sex, presence of acute or chronic comorbidities (i.e., diabetes, COPD, cancer, chronic hepatitis, chronic kidney disease, HIV, neutropenia, and solid organ transplantation), Charlson's Comorbidity Index [21]) and APACHE II score, previous surgery, steroid and/or immunosuppressive therapy ( $\leq 30$ days before BSI onset), and any invasive procedures ( $\leq 72 \mathrm{~h}$ before BSI onset). The isolation of KPC strains from other sites ( $\leq 30$ days) or concomitant infections were also considered. Sepsis or septic shock were evaluated according to the criteria of the International Consensus Definition for Sepsis and Septic shock [22]. Hospitalization variables included nosocomial or healthcare-related infection, days between admission and onset of infection, and total days of hospitalization in the previous year. Treatment variables included antibiotic therapy with ceftazidimeavibactam in monotherapy or combination therapy, antibiogram availability, type and number of drugs, the use of ceftazidime-avibactam as salvage therapy after first-line treatment with other antimicrobials or in first-line therapy [10].

The outcome measured was death, relapse or persistence of infection within 30 days from the first positive blood culture. 
The identification of species was performed with MALDI-TOF mass spectrometry (bioMerieux, Marcy l'Etoile, France), and the detection of KPC was assessed with Genexpert (Cepheid, Sunnyvale, CA, USA). Susceptibility testing was performed by Vitek 2 system (bio-Merieux, Marcy l'Etoile, France) and interpreted according to the EUCAST 2022 definition [23], excluding ceftazidime-avibactam susceptibility, which was determined by MIC Test Strip (Liofilchem, Roseto degli Abruzzi, Italy).

Categorical variables were expressed as absolute numbers and their relative frequencies and compared by the $\chi^{2}$ or Fisher exact test; continuous variables were expressed as median and interquartile range (IQR) and evaluated by the Wilcoxon test and the MannWhitney U test (for no normally distributed variables). Variables that reached a statistical significance $(p<0.05)$ at univariate analysis were analyzed by multivariate logistic regression analysis to identify independent risk factors for mortality. The results obtained were analyzed using the software package SPSS 20.0 (IBM, Armonk, NY, USA).

\section{Conclusions}

In conclusion, CZA showed high efficacy in infections caused by MDR Gram-negative pathogens with limited therapeutic options. This study has some limitations related to its single center, retrospective nature, the statistical heterogeneity, the limited number of patients included in the analysis, the heterogeneity of the isolates and the therapies. Additional data produced by clinical practice are needed to elucidate the role of this molecule in managing infections caused by Gram-negative pathogens with limited therapeutic options.

Author Contributions: Conceptualization: L.B., J.C. and M.D.P.; supervision: F.B., O.C., M.T. and A.G.; formal analysis: L.B., J.C., S.M., M.D.P., P.M., B.C., F.P., S.O., F.G., G.C. and S.C.; resources: E.C. (Emanuele Cocci), R.G.P., E.C. (Elisabetta Cerutti) and O.S.; original draft preparation: L.B., M.G. and M.D.P. All authors have read and agreed to the published version of the manuscript.

Funding: This study was supported by internal funding.

Institutional Review Board Statement: The study was conducted according to the guidelines of the Declaration of Helsinki, and it was approved by the Institutional Review Board of the Azienda Ospedaliero-Universitaria Ospeadali Riuniti Umberto ${ }^{\circ}$-Lancisi-Salesi.

Informed Consent Statement: The Institutional Review Board of the Azienda Ospedaliero-Universitaria Ospeadali Riuniti Umberto $\mathrm{I}^{\circ}$-Lancisi-Salesi granted retrospective access to the data without need for individual informed consent.

Data Availability Statement: Data were collected from the medical case sheets and the laboratory and radiology data, available on the hospital's electronic database.

Conflicts of Interest: The authors declare no conflict of interest.

\section{References}

1. Akova, M. Epidemiology of antimicrobial resistance in bloodstream infections. Virulence 2016, 7, 252-266. [CrossRef] [PubMed]

2. Padmini, N.; Ajilda, A.A.K.; Sivakumar, N.; Selvakumar, G. Extended spectrum $\beta$-lactamase producing Escherichia coli and Klebsiella pneumoniae: Critical tools for antibiotic resistance pattern. J. Basic Microbiol. 2017, 57, 460-470. [CrossRef]

3. Jayol, A.; Nordmann, P.; Poirel, L.; Dubois, V. Ceftazidime/avibactam alone or in combination with aztreonam against colistinresistant and carbapenemase-producing Klebsiella pneumoniae. J. Antimicrob. Chemother. 2018, 73, 542-544. [CrossRef]

4. Liscio, J.L.; Mahoney, M.V.; Hirsch, E.B. Ceftolozane/tazobactam and ceftazidime/avibactam: Two novel $\beta$-lactam/ $\beta$-lactamase inhibitor combination agents for the treatment of resistant Gram-negative bacterial infections. Int. J. Antimicrob. Agents 2015, 46, 266-271. [CrossRef]

5. Behzadi, P.; García-Perdomo, H.A.; Karpiński, T.M.; Issakhanian, L. Metallo-ß-lactamases: A review. Mol. Biol. Rep. 2020, 47, 6281-6294. [CrossRef]

6. Available online: https://www.ema.europa.eu/en/documents/product-information/zavicefta-epar-product-information_en. pdf (accessed on 1 January 2022).

7. Chen, M.; Zhang, M.; Huang, P.; Lin, Q.; Sun, C.; Zeng, H.; Deng, Y. Novel $\beta$-lactam/ $\beta$-lactamase inhibitors versus alternative antibiotics for the treatment of complicated intra-abdominal infection and complicated urinary tract infection: A meta-analysis of randomized controlled trials. Expert Rev. Anti-Infect. Ther. 2018, 16, 111-120. [CrossRef] [PubMed] 
8. Shiber, S.; Yahav, D.; Avni, T.; Leibovici, L.; Paul, M. $\beta$-Lactam/ $\beta$-lactamase inhibitors versus carbapenems for the treatment of sepsis: Systematic review and meta-analysis of randomized controlled trials. J. Antimicrob. Chemother. 2015, 70, 41-47. [CrossRef]

9. Issakhanian, L.; Behzadi, P. Antimicrobial Agents and Urinary Tract Infections. Curr. Pharm. Des. 2019, 25, 1409-1423. [CrossRef] [PubMed]

10. Tumbarello, M.; Trecarichi, E.M.; Corona, A.; De Rosa, F.G.; Bassetti, M.; Mussini, C.; Menichetti, F.; Viscoli, C. Efficacy of Ceftazidime-Avibactam Salvage Therapy in Patients With Infections Caused by Klebsiella pneumoniae Carbapenemaseproducing K. pneumoniae. Clin. Infect. Dis. 2019, 68, 355-364. [CrossRef]

11. Mazuski, J.E.; Wagenlehner, F.; Torres, A.; Carmeli, Y.; Chow, J.W.; Wajsbrot, D.; Stone, G.G.; Irani, P.; Bharucha, D.; Cheng, K.; et al. Clinical and Microbiological Outcomes of Ceftazidime-Avibactam Treatment in Adults with Gram-Negative Bacteremia: A Subset Analysis from the Phase 3 Clinical Trial Program. Infect. Dis. Ther. 2021, 10, 2399-2414. [CrossRef]

12. Shields, R.K.; Nguyen, M.H.; Chen, L.; Press, E.G.; Potoski, B.A.; Marini, R.V.; Doi, Y.; Kreiswirth, B.N.; Clancy, C.J. CeftazidimeAvibactam Is Superior to Other Treatment Regimens against Carbapenem-Resistant Klebsiella pneumoniae Bacteremia. Antimicrob. Agents Chemother. 2017, 61, e00883-17. [CrossRef]

13. Castón, J.J.; Gallo, M.; García, M.; Cano, A.; Escribano, A.; Machuca, I.; Gracia-Aufinger, I.; Guzman-Puche, J.; Pérez-Nadales, E.; Recio, M.; et al. Ceftazidime-avibactam in the treatment of infections caused by KPC-producing Klebsiella pneumoniae: Factors associated with clinical efficacy in a single-center cohort. Int. J. Antimicrob. Agents 2020, 56, 106075. [CrossRef]

14. Kohler, P.P.; Volling, C.; Green, K.; Uleryk, E.M.; Shah, P.S.; McGeer, A. Carbapenem resistance, initial antibiotic therapy, and mortality in Klebsiella pneumoniae bacteremia: A systematic review and meta-analysis. Infect. Control Hosp. Epidemiol. 2017, 38, 1319-1328. [CrossRef] [PubMed]

15. Falagas, M.E.; Tansarli, G.S.; Karageorgopoulos, D.E.; Vardakas, K.Z. Deaths attributable to carbapenem-resistant Enterobacteriaceae infections. Emerg. Infect. Dis. 2014, 20, 1170-1175. [CrossRef] [PubMed]

16. Benattar, Y.D.; Omar, M.; Zusman, O.; Yahav, D.; Zak-Doron, Y.; Altunin, S.; Elbaz, M.; Daitch, V.; Granot, M.; Leibovici, L.; et al. The effectiveness and safety of high-dose colistin: Prospective cohort study. Clin. Infect. Dis. 2016, 63, 1605-1612. [CrossRef]

17. Barchiesi, F.; Montalti, R.; Castelli, P.; Nicolini, D.; Staffolani, S.; Mocchegiani, F.; Fiorentini, A.; Manso, E.; Vivarelli, M. Carbapenem-resistant Klebsiella pneumoniae influences the outcome of early infections in liver transplant recipients. BMC Infect. Dis. 2016, 16, 538. [CrossRef] [PubMed]

18. Clancy, C.J.; Chen, L.; Shields, R.K.; Zhao, Y.; Cheng, S.; Chavda, K.D.; Hao, B.; Hong, J.H.; Doi, Y.; Kwak, E.J. Epidemiology and molecular characterization of bacteremia due to carbapenem-resistant Klebsiella pneumoniae in transplant recipients. Am. J. Transplant. 2013, 13, 2619-2633. [CrossRef]

19. Villegas, M.V.; Pallares, C.J.; Escandón-Vargas, K. Characterization and clinical impact of bloodstream infection caused by carbapenemase-producing Enterobacteriaceae in seven Latin American Countries. PLoS ONE 2016, 11, e0154092. [CrossRef]

20. Chen, F.; Zhong, H.; Yang, T.; Shen, C.; Deng, Y.; Han, L.; Chen, X.; Zhang, H.; Qian, Y. Ceftazidime-Avibactam as Salvage Treatment for Infections Due to Carbapenem-Resistant Klebsiella pneumoniae in Liver Transplantation Recipients. Infect. Drug Resist. 2021, 14, 5603-5612. [CrossRef]

21. Charlson, M.E.; Pompei, P.; Ales, K.L.; MacKenzie, C.R. A new method of classifying prognostic comorbidity in longitudinal studies: Development and validation. J. Chronic Dis. 1987, 40, 373-383. [CrossRef]

22. Singer, M.; Deutschman, C.S.; Seymour, C.W.; Shankar-Hari, M.; Annane, D.; Bauer, M.; Bellomo, R.; Bernard, G.R.; Chiche, J.D.; Coopersmith, C.M.; et al. The Third International Consensus Definitions for Sepsis and Septic Shock (Sepsis-3). JAMA 2016, 315, 801-810. [CrossRef] [PubMed]

23. The European Committee on Antimicrobial Susceptibility Testing. Breakpoint Tables for Interpretation of MICs and Zone Diameters. Version 12.0. 2022. Available online: https://www.eucast.org/fileadmin/src/media/PDFs/EUCAST_files/Breakpoint_ tables/v_12.0_Breakpoint_Tables.pdf (accessed on 1 January 2022). 